\title{
Conflicts as signals: bridging the gap between conflict detection and cognitive control
}

\author{
Birgit Stürmer $\cdot$ Werner Sommer $\cdot$ Peter Frensch
}

Published online: 24 December 2008

(C) Springer-Verlag 2008

"Cognitive conflicts" are said to occur when several incompatible action tendencies or motivations co-exist in our cognitive system. The processing problems arising from cognitive conflicts are often assumed to reflect basic principles of the cognitive architecture. "Cognitive control" comes into play whenever conflicts need to be resolved to guarantee flawless behavior; how exactly cognitive conflicts are resolved is one of the major challenges that current models of cognitive control are facing.

Most of the existing research on conflicts in the human information processing system has focused on the processes that elicit conflicts and the processes that monitor and resolve conflict. Importantly, very little research has tried to understand the consequences of conflicts for subsequent information processing. In contrast, all the articles in this Special Issue reflect the belief that conflicts are functional and that they can be viewed as signals that trigger information processing within the cognitive system and lead to adaptation of the system configuration. Thus, the research described in this Special Issue has begun to unravel the processes that mediate between the conflict signal indicating discrepancies or problems in the information processing system and subsequent optimization mechanisms. Hence, conflict-driven behavioral optimization becomes the research focus.

One seminal theory that drew our attention to the role of conflicts and their subsequent adaptation processes is the conflict monitoring account described by Botvinick and colleagues (Botvinick, Braver, Barch, Carter, \& Cohen,

B. Stürmer $(\bowtie) \cdot$ W. Sommer $\cdot$ P. Frensch

Department of Psychology, Humboldt-Universität zu Berlin,

Rudower Chaussee 18, 12489 Berlin, Germany

e-mail: birgit.stuermer@cms.hu-berlin.de
2001; Botvinick, Cohen, \& Carter, 2004). In the Botvinick et al. model, the dorsal anterior cingulate cortex (ACC) monitors the occurrence of potential conflicts. Detected conflicts then trigger resolution processes in the prefrontal cortex. Only recently has Botvinick (2007) extended his account to the ACC by proposing that the ACC is involved in biasing behavior away from tasks or strategies that have been effortful in the past. Here, conflicts can be viewed as signals that trigger subsequent processes with the objective of avoiding imminent conflicts. Conflicts are, thus, seen to trigger proactive control strategies (Braver, Gray, Burgess, 2007) that prevent conflicts from occurring in the future.

Although adaptation after conflict is corroborated by empirical findings in different domains of cognitive psychology the origins of the adaptive consequences of conflicts are still under debate. This Special Issue, therefore, gives an overview of various approaches that highlight different aspects of adaptive conflict control processing. Twelve contributions to this Special Issue deal with different types of conflicts as well as different adaptation processes after conflicts and center around five central questions. First, do different types of conflicts lead to different adaptive processes or do different conflicts always lead to similar adaptive processes? Second, what exactly is the relation between conflict-induced adaptation and the topdown regulation of information processing? Here, concepts such as working memory and fluid intelligence might play important roles. Adaptive processing is, of course, closely linked to the anticipation of the outcome of future actions. As anticipation processes may not only be influenced by cognitive consequences of conflicts but also by affective evaluation, third, one may ask, about the role of affect in adaptive processing that follows conflicts. Fourth and fifth, are there meaningful and stable interindividual differences and developmental patterns in the types of signals that are 
generated by conflicts and the adaptive processing that follows conflicts?

The collection of articles that comprises the Special Issue is based on contributions to workshops that were held in Berlin, Germany on 11-12 May 2007, and in Binz, Germany, on 21-23 September 2007. We are very grateful to the participants of the workshops and, in particular, to the authors of the present articles for shaping and clarifying our research questions and for their willingness to contribute an extended version of the talks they presented at the workshop to the Special Issue.

In the first article, Brown, in testing predictions of his error likelihood computational model, investigates whether conflict and error likelihood produce distinct cognitive control effects. To this end, the change signal task (Brown \& Braver, 2005) was modified to manipulate the error likelihood of too fast and too slow responses in conditions with and without conflict. The results suggest that adaptation due to error anticipation and conflict-related adaptation is based on distinct processes and, hence, suggest multiple cognitive control processes.

Kehrer, Kraft, Irlbacher, Koch, Hagendorf, Kathmann, and Brandt provide further evidence that solving conflicts affects subsequent information processing from a visual selection task. Here, negative priming seems to occur as a consequence of experiencing a conflict in the previous trial. Visual selection demands were manipulated and resulted in reduced negative priming in events that called for high loads in cognitive control. Event-related potentials were measured to investigate the role of visual spatial attention mechanisms in the context of conflict processing. Similar to other interference studies, conflict elicited in negative priming trials was accompanied by an enlarged frontocentral N2 amplitude that was even increased when cognitive control demands were high in difficult visual selection trials.

Keye, Wilhelm, Oberauer, and Ravenzwaaij focus on interindividual differences in conflict control and sequencedependent adaptation to conflict. The authors' measurement models did not capture a general factor for performance in the Simon and Eriksen tasks. Moreover, working memory measures correlated consistently with factors of general RT performance but not with any conflict factors. Hence, the view that working memory is related to the ability to control attention and thus reduces conflict (Heitz \& Engle, 2007) was not supported. Sequence-dependent conflict adaptation was only related to working memory performance in the Simon task but not in the Eriksen task. The strong task-specificity of their conflict and control measures obtained through their measurement models are also troublesome for theories assuming that cognitive control reflects a general ability that can be linked to working memory capacity (Kane et al., 2004).
Li, Hämmerer, Müller, Hommel, and Lindenberger investigate developmental aspects of the relation between cognitive control and relate cognitive conflict to fluid intelligence measured by variables such as cognitive speed, memory, and reasoning. The lifespan age gradient of conflict cost parallels those gradients obtained for processing fluctuation and fluid intelligence. Individuals at both ends of the lifespan display a greater amount of processing fluctuation and at the same time a larger amount of conflict cost and a lower level of fluid intelligence. After controlling for chronological age and baseline processing speed, conflict cost continues to correlate significantly with fluid intelligence in adulthood and old age and with processing fluctuation in old age. The relation between processing fluctuation and conflict cost in old age lends further support for the neuromodulation of neuronal noise theory of cognitive aging (Li, Lindenberger, \& Sikström, 2001) as well as for theories of dopaminergic modulation of conflict monitoring (Montague, Hyman, \& Cohen, 2004).

Kerns reviews previous research on three types of processing conflict, namely, response conflict, retrieval conflict, and emotional conflict. Common to all three types of processing conflicts is the involvement of active maintenance of task-critical information and task goals in conflict resolution. Kerns suggests that such a maintenance process is a task-general mechanism of cognitive control that is hampered in neuropsychatric disorders of control such as schizophrenia.

Mayr and Awh replicate Kerns et al. 2004 finding of inter-trial interference adaptation in the Stroop paradigm. However, they can show that the interference reduction is conflict-driven only during an early phase of the experiment, whereas after a first practice phase inter-trial interference adaptation is completely due to direct item repetitions. Mayr and Awh, therefore, assume that conflict-driven adaptation occurs only in situations when tasks are processed in a top-down manner.

Alpay, Görke, and Stürmer investigate whether sequence-dependent interference adaptation can be accounted for by strategic executive control. To this end, precues informed about upcoming conflicts. Cue-induced adaptation did not override conflict-driven intertrial adjustments. Their findings are, hence, in favor of two distinguishable adjustment processes and thereby challenge the conflict monitoring account described by Botvinick et al. (2004) proposing that conflict-driven adjustments are related to top-down executive control processes that provoke a strategic shift in task demands.

Adaptive consequences of conflict are, however, not restricted to cognitive interference tasks. Haider and Frensch investigate conflicts between individuals' expectations and their actual behaviors in an incidental learning situation. The authors can show that this particular type of 
conflict is associated with verbalizable knowledge of the incidentally experienced environmental regularity and that the ability to verbalize incidentally acquired knowledge, in turn, is related to strategic optimization. Thus, conflicts between expectations and actual behaviors lead to topdown initiated modifications of task strategies.

Botvinick and Rosen manipulate mental effort and show that the anticipatory skin conductance response (SCR) - a parameter related to responses of the autonomous nervous system-increases prior to actions drawing on a high level of cognitive demand. This finding indicates that the necessity for later effortful cognitive control is already anticipated during action selection.

Schacht, Nigbur, and Sommer investigate affective implications of cognitive interference. According to SCR, corrugator muscle activity, and the startle blink response, interference in NoGo trials seems not to be aversive. NoGos rather appear to be obstructive to the response goal and less action-relevant than Go trials.

Segalowitz and Dywan review interindividual differences as well as developmental patterns of processing conflicting information. The authors focus on the error-related negativity (ERN) as an electrophysiological marker of processing erroneous responses that is subject to developmental change across the lifespan as well as to individual differences in personality, affect, and autonomic responsivity. As the ERN is closely related to ACC, such influences imply the need to integrate neurovisceral/affective and cognitive domains and personality factors in any model of ACC function.

Klonek, Tamm, Hofmann, and Jacobs contrast predictions of the conflict monitoring account (Botvinick et al., 2001) with those of the Multiple-Read-Out-Model (MROM; Jacobs and Grainger 1994). To this end, a word stem completion task was conducted and word stems with multiple completions were compared against word stems with only one possible completion. According to the conflict monitoring account, word stems associated with multiple completions should lead to crosstalk and conflict due to the underdetermined responding situation. In contrast, MROM predicts a high level of general lexical activity for word-stems with many completions, indicating a higher stimulus familiarity because these stems are more probable to be read. A challenge to the conflict monitoring account was the missing conflict-related N2 for word stems with multiple completions that usually accompanies cognitive interference (Yeung, Botvinick, Cohen, 2004).
Together, the collection of articles contained in this Special Issue provides an overview of some of the work that is currently conducted in the field to disentangle the mechanisms underlying conflict monitoring, conflict resolution, and cognitive control. Although the scope of the contributions is necessarily limited, we believe that the articles are useful and that the approach they are reflecting has the potential to become highly fruitful, both theoretically and empirically. It may well be time to consider conflicts not as nuisances but as "signals" that have a function in the human information processing system and act to trigger subsequent processing with the implicit or explicit goal to optimize the performance of the system.

\section{References}

Botvinick, M. M. (2007). Conflict monitoring and decision making: reconciling two perspectives on anterior cingulate function. Conflict Affective and Behavioral Neuroscience, 7(4), 356-366.

Botvinick, M. M., Braver, T. S., Barch, D. M., Carter, C. S., \& Cohen, J. D. (2001). Conflict monitoring and cognitive control. Psychological Review, 108, 624-652.

Botvinick, M. M., Cohen, J. D., \& Carter, C. S. (2004). Conflict monitoring and anterior cingulate cortex: An update. Trends in Cognitive Sciences, 8(12), 539-546.

Braver, T. S., Gray, J. R., \& Burgess, G. C. (2007). Explaining the many varieties of working memory variation: Dual mechanisms of cognitive control. In A. Conway, C. Jarrold, M. Kane, A. Miyake, \& J. Towse (Eds.), Variation of working memory. Oxford: Oxford University Press.

Brown, J., \& Braver, T. (2005). Learned predictions of error likelihood in the anterior cingulate cortex. Science, 307, 1118-1121.

Heitz, R. P., \& Engle, R. W. (2007). Focusing the spotlight: Individual differences in visual attention control. Journal of Experimental Psychology: General, 136(2), 217-240.

Jacobs, A. M., \& Grainger, J. (1994). Models of visual word recognition-sampling the state of the art. Journal of Experimental Psychology, 20(6), 1311-1334.

Kane, M. J., Hambrick, D. Z., Tuholski, S. W., Wilhelm, O., Payne, T. W., \& Engle, R. W. (2004). The generality of working memory capacity: A latent-variable approach to verbal and visuospatial memory span and reasoning. Journal of Experimental Psychology: General, 133, 189-217.

Kerns, J. G., Cohen, J. D., MacDonald, A. W., Cho, R. Y., Stenger, V. A., \& Carter, C. S. (2004). Anterior cingulate conflict monitoring and adjustments in control. Science, 303, 1023-1026.

Li, S.-C., Lindenberger, U., \& Sikström, S. (2001). Aging cognition: from neuromodulation to representation. Trends in Cognitive Sciences, 5, 479-486.

Montague, P. R., Hyman, S. E., \& Cohen, J. D. (2004). Computational roles for dopamine in behavioral control. Nature, 431, 760-767.

Yeung, N., Botvinick, M. M., \& Cohen, J. D. (2004). The neural basis of error detection: Conflict monitoring and the error-related negativity. Psychological Review, 111(4), 931-959. 